\title{
Syphilis Mimicking Other Dermatological Diseases: Reactive Arthritis and Mucha-Habermann Disease
}

\author{
Sueli Coelho da Silva Carneiro ${ }^{a}$ Rodrigo Pirmez ${ }^{a}$ \\ Taciana Rocha de Hollanda ${ }^{a}$ Tullia Cuzzi ${ }^{b}$ Marcia Ramos-e-Silva ${ }^{a}$ \\ a Sector of Dermatology and Post-Graduation Course, and ' $S$ Sector of Pathology, \\ HUCFF/UFRJ, and School of Medicine, Federal University of Rio de Janeiro, \\ Rio de Janeiro, Brazil
}

\section{Key Words}

Syphilis · Reactive arthritis · Reiter syndrome $\cdot$ Mucha-Habermann disease

\begin{abstract}
The authors present two cases of syphilis: one mimicking reactive arthritis and the other Mucha-Habermann disease. Both reports illustrate syphilis as 'the great imitator', a description given by Sir William Osler, and call attention to the strong need for awareness among physicians of all specialties, especially the younger ones, who are not used to seeing this increasingly prevalent disease, as it once was in the past.
\end{abstract}

\section{Introduction}

Syphilis is a chronic systemic infectious disease caused by Treponema pallidum, a microaerophilic spirochete, whose transmission occurs mainly by sexual contact. Its course is characterized by a multistage evolution, in a total of 4 distinct clinical stages in cases of nontreated disease, in which symptomatic phases alternate with periods of latency [1]. The myriad of possible clinical manifestations often represent a great challenge, a fact that led Sir William Osler to label it as 'the great imitator'.

In this report, we present 2 cases in which the clinical manifestations of the patients initially simulated other dermatologic diseases, giving the impression of Mucha-Habermann disease (pityriasis lichenoides et varioliformis acuta), in the first case and Reiter's syndrome (reactive arthritis), in the second. 


\section{Case Reports}

\section{Patient 1}

Male patient, 18 years old, white, single, student, born in and resident of Rio de Janeiro. He presented with a 1-month history of cutaneous lesions, jaundice, darkened urine and acholic stools. The patient admitted sexual relations without protection.

There was lesional polymorphism with lesions varying from erythematous papules and pustules to ulceronecrotic lesions with disseminated crusts on the face, trunk and limbs (fig. 1). Physical examination revealed jaundice $(++/ 4+)$ and bilateral inguinal lymphadenopathy. The exams showed positive serology for hepatitis B and alteration of the hepatic function tests. VDRL was 1/64 and anti-HIV negative. The histopathological features of the skin lesion biopsy were compatible with syphilis. The initial impression of cutaneous vasculitis was not confirmed and clinical data, serologic tests and histologic findings led to the diagnosis of early malignant syphilis in an immunocompetent patient. He was treated with penicillin benzathine 2,400,000 - 2 doses with a 7-day interval.

\section{Patient 2}

Male patient, 19 years old, single, student, born in and resident of Rio de Janeiro, presenting with conjunctival hyperemia and eyelid swelling on the right side for almost 2 years, which initially improved with steroid eye drops. Later, there was progressive loss of visual acuity and onset of erythematous scaly lesions on the buttocks, palms and soles, besides erosions on the glans and inside the oral cavity (fig. 2, fig. 3).

During the 6-month period preceding the medical consultation, arthralgia appeared on the knees, left sacroiliac articulation and elbows, accompanied by headache. The ophthalmological examination revealed panuveitis and the dermatological examination revealed keratoderma palmoplantaris, diffuse erythema on the trunk, scrotum erythema and erosion, circular residual violaceous erythema on the glans, anonychia and onychodystrophy, which hinted at the diagnosis of reactive arthritis. The complementary tests were positive for HIV and syphilis (VDRL of 1/128).

\section{Discussion}

Syphilis is characterized not only for evolving in distinct clinical stages, but also for having the ability to mimic a wide variety of diseases, in each of its phases.

In primary syphilis, the lesion appears on average 21 days after the infection. Localized erythema develops at the site of inoculation, which evolves into hardened and painless papules. Classically, after the surface necrosis, the typical well-circumscribed ulceration develops, with hardened borders and clear base - the chancre - associated with regional adenopathy [2]. Chancres must always be included in the differential diagnosis of any genital ulcer [1]. Primary syphilis, however, may present atypical morphology, location and symptoms, causing diagnostic difficulties, which results in only $30-40 \%$ of patients being diagnosed in the primary stage [3].

Extragenital chancres can occur in any mucocutaneous surface exposed to the infection, but are more common in the oral cavity and anal region [3]. Any ulcerated nodular lesion associated to lymph adenopathy should lead to the suspicion of primary syphilis. Differential diagnosis must be made with other infections, including tularemia; cat-scratch disease; sporotrichosis; mycobacteriosis; leishmaniasis and staphylococcal lymphangitis; and with granulomatous diseases and neoplasms with nodal metastasis. In the anal region, it is rarely 
restricted only to a nodular hardening and can be accompanied by fissures, which leads to confusion with hemorrhoids, anal fissures or even neoplasms [1]. Features vary according to the number of inoculated spirochetes, concomitant use of antibiotics, presence of coinfections, and the patient's immune state $[1,4]$.

The secondary stage results from the hematogenous and lymphatic dissemination and multiplication of the microorganism in different tissues. Recurring activity is characteristic of the disease with systemic and mucocutaneous manifestations [2]. Not all patients present the classic signs and symptoms. These may be subtle, transient and pass unnoticed; or so severe as to require hospitalization [5]. However, over $90 \%$ of patients present rash, which is almost always characteristic [4]. At the beginning, there is a macular rash with discrete pink, non-scaling, oval lesions - the syphilitic roseola -, predominantly on the trunk and upper flexor areas of limbs. Then, the lesions may develop into a papular-macular, papulardesquamative, lenticular, corymbose, nodular, annular, follicular, pustular or impetiginous aspect [5]. They can be generalized or grouped and located at precise spots [1]. Secondary syphilis polymorphism depends entirely on the intensity of the inflammatory infiltrate, level of cutaneous vascular involvement and the resulting ischemia [6].

When papular lesions are pruritic and lichenoid, it may be difficult to differentiate from lichen planus [7]. Annular lesions may also resemble annular granuloma, pityriasis rosea and dermatophytosis [8]. Where there is a hyper keratotic component, the resulting lesions are indistinguishable from psoriasis [5, 8]. Hyperkeratotic plaques on the soles give the impression of calluses and, when desquamative, can mimic tinea pedis [1,5]. The differential diagnosis of nodular syphilis includes systemic mycosis, Kaposi's sarcoma, bacillary angiomatosis, foreign body granuloma type, lymphoma, pseudolymphoma, leprosy, sarcoidosis, and halogenoderma. Secondary syphilis with pustular lesions can also lead to the erroneous diagnosis of pustular acne $[8,9]$.

Mucosal involvement can be part of the condition, consisting mainly of mucous patches, pharyngitis and condyloma lata [5]. The latter may be confused with condyloma acuminata [8]. The involvement of the tongue is rare and can simulate oral hairy leukoplakia [10].

Hair can be affected on the scalp, eyebrows and beard, and diffuse pattern alopecia increases the index of suspicion for the disease [11]. The latter may be similar to alopecia areata, neoplastic alopecia, tinea capitis or trichotillomania [12]. Ungual disorders include fissures, Beau lines, onycholysis, onychomadesis, onychogryphosis, nail pitting and others $[1,6]$.

Completing the picture, general symptoms may occur and rarely hepatitis, periostitis, arthritis, gastritis, meningitis and uveitis [3]. In the second case reported here, the ocular and articular involvements associated with the mucocutaneous lesions led to a first diagnostic impression of reactive arthritis. This syndrome is characterized by the classical triad of arthritis, urethritis and conjunctivitis, after urethral or gastrointestinal infection [13]. Despite conjunctivitis being classically described in the syndrome, there are other associated ocular involvements, such as iritis and uveitis, the latter being diagnosed in our patient. That young man also presented, at the dermatological examination, keratoderma palmoplantaris and erosions on the glans, simulating, respectively, keratoderma blenorrhagica and circinate balanitis, and erosive lesions of the oral cavity. All these findings have been described in up to $20 \%$ of patients with reactive arthritis [13]. The ungual disorders presented are also described in the syndrome.

A variant of secondary syphilis known as early malignant syphilis is characterized by a fast progression of papules, plaques, nodules, and polymorphic vesicular pustules with tendency to ulceration, covered with several layers of thick crusts, acquiring a rupioid aspect [6]. The rash may be accompanied by toxemia and sometimes hepatitis [7]. This clinical 
da Silva Carneiro et al.: Syphilis Mimicking Other Dermatological Diseases: Reactive Arthritis and Mucha-Habermann Disease

picture is compatible with the one presented by patient 1 . However, at first glance, the acute onset and the exuberant features of the lesions led to the diagnostic hypothesis of pityriasis lichenoides et varioliformis acuta. Also known as Mucha-Habermann disease, this disease is characterized by the appearance of generalized erythematous-purpuric papules that evolve with necrosis. The condition is marked by spontaneous remission of lesions and frequent recurrences [14]. The lesions' histopathological analysis excluded that hypothesis in favor of syphilis.

Finally, untreated syphilis evolves into a third form, very rare nowadays, with a variety of manifestations that occur months to years after the beginning of the infection. This stage is characterized by the presence of a small number of Treponema, but with high cellular immune reactivity against this agent. Microorganisms can invade different systems and lead to injury by a delayed type of hypersensitivity reaction [2].

\section{Conclusion}

Syphilis presents a highly variable clinical course and wide diversity of manifestations, which has made it known as the great mimicker. Its ability to simulate various other diseases often causes its diagnosis to be a challenge. Its clinical exuberance is often no longer recognized, especially by younger physicians. However, the lack of diagnosis of this quite prevalent disease can entail serious consequences for the patient. Thus, it is of considerable importance that dermatologists and other specialists be familiarized with the many manifestations of syphilis and start considering it more often among their diagnostic hypotheses.

\section{References}

1 Lautenschlager S: Cutaneous manifestations of syphilis - recognition and management. Am J Clin Dermatol 2006;7:291-304.

2 Stary A: Sexually transmitted infections; in Bolognia JL, Jorizzo JL, Rapini RP (eds): Dermatology, ed 2. London, Mosby, 2008, pp 1239-1261.

-3 Lautenschlager S: Diagnosis of syphilis: clinical and laboratory problems. J Dtsch Dermatol Ges 2006;4:1058-1075.

4 Golden MR, Marra CM, Holmes KK: Update on syphilis: resurgence of an old problem. JAMA 2003;290:15101514.

5 Sanchez MR: Syphilis; in Wolff K, Goldsmith LA, Katz SI, Gilchrest BA, Paller AS, Leffell DJ (eds): Fitzpatrick's Dermatology in General Medicine, ed 7. New York, McGraw-Hill, 2008, pp 1955-1977.

6 Dourmishev LA, Dourmishev AL: Syphilis: uncommon presentations in adults. Clin Dermatol 2005;23:555564.

7 Tang MB, Yosipovitch G, Tan SH: Secondary syphilis presenting as a lichen planus-like rash. J Eur Acad Dermatol Venereol 2004;18:185-187.

8 Domantay-Apostol GP, Handog EB, Gabriel MT: Syphilis: the international challenge of the great imitator. Dermatol Clin 2008;26:191-202.

-9 Moon HS, Park K, Lee JH, Son SJ: A nodular syphilid presenting as a pseudolymphoma: mimicking a cutaneous marginal zone B-cell lymphoma. Am J Dermatopathol 2009;31:846-848.

10 Aquilina C, Viraben R, Denis P: Secondary syphilis simulating oral hairy leukoplakia. J Am Acad Dermatol 2003;49:749-751.

11 Zampese Benvenuto-Andrade C, Cunha V: Doenças sexualmente transmissíveis e treponematoses nãosexuais; in Ramos-e-Silva M, Castro MCR (eds): Fundamentos de Dermatologia, Rio de Janeiro, Atheneu, 2010, pp 943-977.

12 Bi MY, Cohen PR, Robinson FW, Gray JM: Alopecia syphilitica-report of a patient with secondary syphilis presenting as moth-eaten alopecia and a review of its common mimickers. Dermatol Online J 2009;15:6.

13 Fougerousse AC, Bonnel S, Peron L, Pessey F, Carsuzaa F: Arthrite réactionnelle (syndrome de FiessingerLeroy-Reiter). Ann Dermatol Venereol 2010;137:425-426.

14 Carneiro SCS, Azulay-Abulafia L, Azulay DR: Sífilis, in Azulay RD, Azulay DR (eds): Dermatologia, ed 4. Rio de Janeiro, Guanabara Koogan, 2006, pp 107-120. 


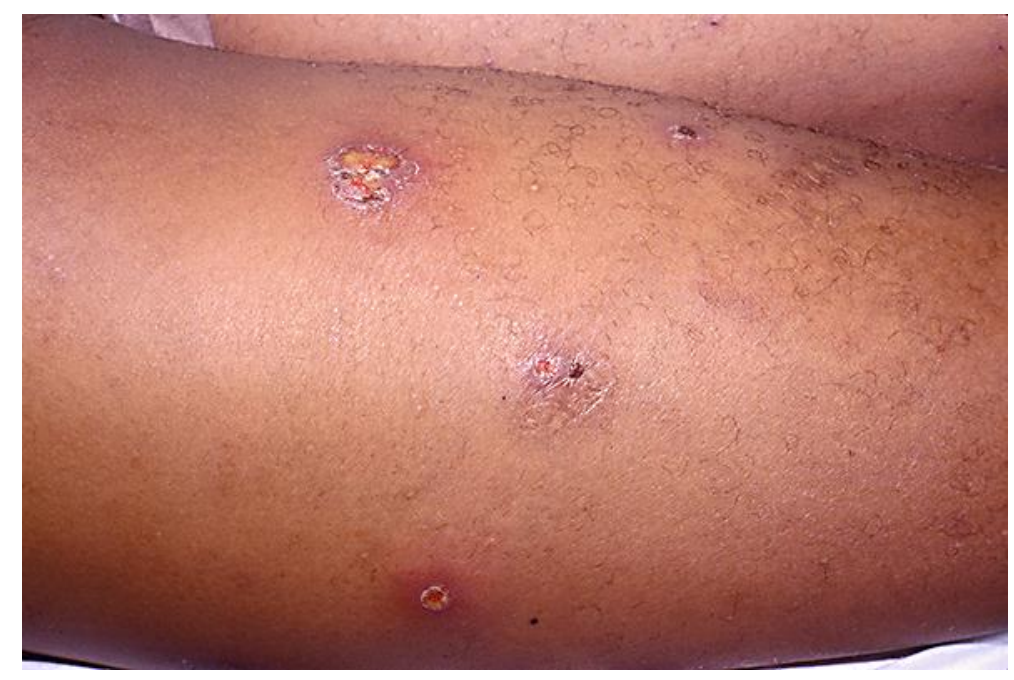

Fig. 1. Patient 1: Necrotic lesion of right thigh.

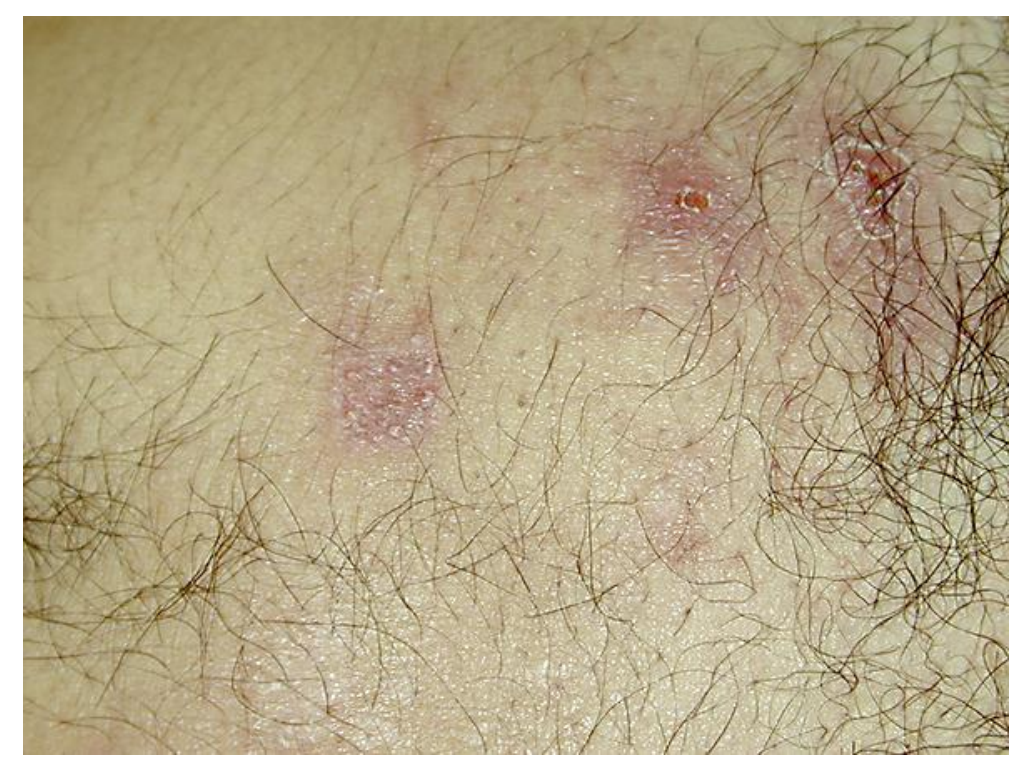

Fig. 2. Patient 2: Erythematous and eroded lesions on the chest. 
da Silva Carneiro et al.: Syphilis Mimicking Other Dermatological Diseases:

Reactive Arthritis and Mucha-Habermann Disease

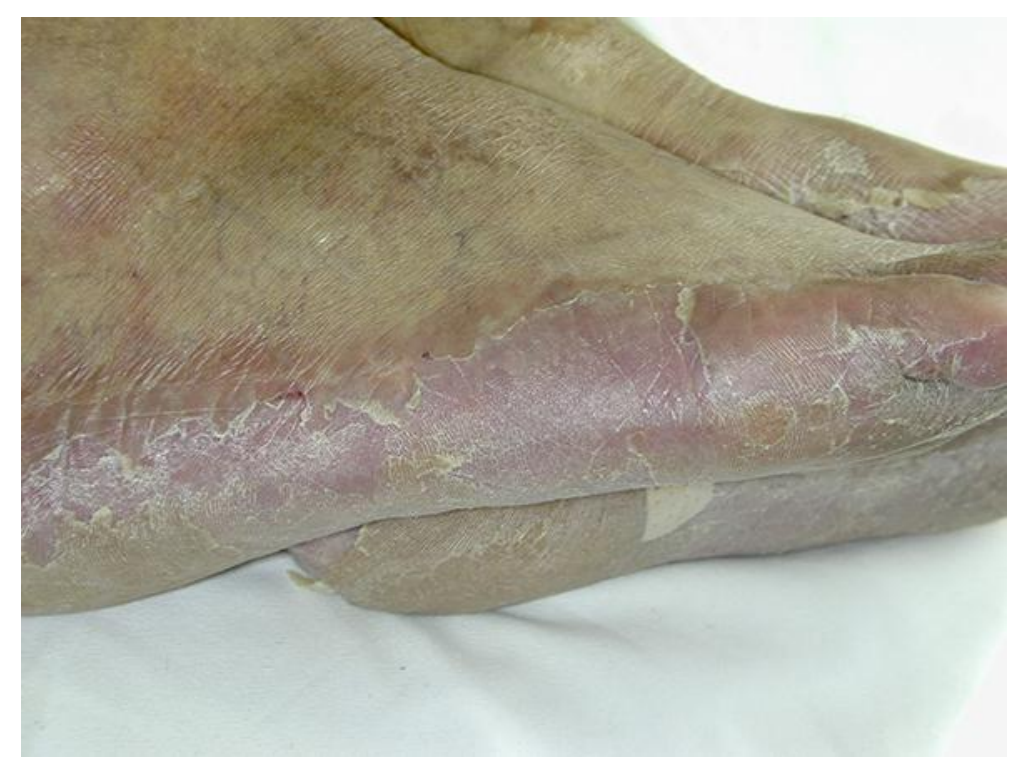

Fig. 3. Patient 2: Plantar hyperkeratosis. 